\section{Correlation between PARP-1 immunoreactivity and cytomorphological features of parthanatos, a specific cellular death in breast cancer cells}

\author{
P. Donizy, ${ }^{1}$ A. Halon, ${ }^{1}$ P. Surowiak, ${ }^{2}$ \\ G. Pietrzyk, ${ }^{3}$ C. Kozyra, ${ }^{4}$ R. Matkowski ${ }^{5,6}$ \\ ${ }^{1}$ Department of Pathomorphology and \\ Oncological Cytology, Wroclaw Medical \\ University, Wroclaw \\ 2Department of Histology and \\ Embryology, Wroclaw Medical University \\ ${ }^{3}$ Laboratory of Mammotomic Biopsy, $4^{\text {th }}$ \\ Military Hospital, Wroclaw \\ ${ }^{4}$ Department of Statistics, Wroclaw \\ University of Economics \\ ${ }^{5}$ Department of Oncology and Division of \\ Surgical Oncology, Wroclaw Medical \\ University \\ 'Lower Silesian Oncology Centre, \\ Wroclaw, Poland
}

\begin{abstract}
In parthanatos, a PARP-1 (poly (ADP-ribose) polymerase 1)-mediated cell death, dissipation of mitochondrial membrane potential, largescale DNA fragmentation and chromatin condensation were observed. In contrast to apoptosis, it does not cause apoptotic bodies formation. Although PARP-1-mediated cell death presents loss of membrane integrity similar to necrosis, it does not induce cell swelling. The purpose of the study was to correlate the immunohistochemical parameters of PARP-1 reactivity and the selected cytomorphological features of parthanatos: the lack of apoptotic bodies and the absence of necrosis in breast cancer (BC) specimens. Immunohisto chemistry for PARP-1 was performed on 83 BC specimens. Correlations between parameters of PARP-1 expression and sub-cellular localisation and the presence of apoptotic bodies and necrosis were evaluated. High expression of PARP-1 (immunoreactive score $\geq 6$ ) was associated with the lack of apoptotic bodies $(\mathrm{P}=0.013)$ and with the absence of necrosis $(\mathrm{P}=0.002)$. The presence of apoptotic bodies was correlated with re-distribution of PARP-1 from the nucleus to cytoplasm in $\mathrm{BC}$ cells $(\mathrm{P}=0.029)$. Additionally, a tendency was observed between necrosis and loss of nuclear PARP-1 expression ( $\mathrm{P}=0.049)$. Our study suggests that PARP-1 may play a crucial role in induction and regulation of specific type of cellular death called parthanatos.
\end{abstract}

\section{Introduction}

PARP-1 has an important role in DNA damage repair and maintaining genome integrity by repairing DNA single strand breaks (SSBs) by base excision repair (BER). It is also involved in chromatin remodelling, regulation of transcription, proliferation processes and differentiation as well as specific cell death called parthanatos. The so-called damage sensor model is proposed in which activated PARP-1 identifies DNA breaks and temporarily binds to the ends of the damaged DNA. ${ }^{1-3}$ PARP-1's enzymatic activity can increase 500fold on its binding to the damaged DNA, which results in quick elongation of PAR chain. ${ }^{4}$ ADPribose polymers situated on PARP-1 molecules attached to the damaged DNA function as specific enticements which facilitate repair by recruiting repair enzymes to the site of damage. Additionally, ADP-ribosylation of XRCC1 (vital for BER) greatly enhances its affinity for BER pathway main enzymes, i.e. DNA ligase III and AP endonuclease., ${ }^{2,5}$

Current knowledge and results of recent studies suggest that the initiation of death cell called parthanatos is closely related with the accumulation of PAR in response to DNA damage. ${ }^{6}$ The polymers function as specific signals and cytophysiological messengers informing about DNA damage that is too extensive to be repaired by the cell itself. Their excess induces parthanatos to prevent and protect against further loss of energy for ineffective DNA repair.,

PARP-1-mediated cell death is morphologically and molecularly distinct from other known forms of cell death such as apoptosis, necrosis or autophagy. Parthanatos has been shown to involve mitochondrial transmembrane potential dissipation, chromatin condensation and large DNA fragmentation. ${ }^{8}$ Unlike apoptosis, it does not cause apoptotic body formation or small scale DNA fragmentation. In vitro experiments with cell lines and caspase inhibitors (z-VAD-fmk, boc-aspartyl-fmk) have conclusively confirmed that the process is caspase-independent and it is not regulated by Bcl-2 proteins. ${ }^{5,9}$ It is worth noting that PARP1-mediated cell death involves loss of membrane integrity similar to necrosis, yet it does not induce cell swelling. ${ }^{10}$ Parthanatos is distinct also from autophagy as it does not involve autophagic vacuoles formation or lysosomal degradation. ${ }^{11,12}$

PARP-1 was widely examined in some types of human tumors, ${ }^{13,14}$ but it must be stressed that there are no reports that would describe cytomorphological features of parthanatos in clinical material obtained from breast cancer (BC) patients in correlation with overexpression of PARP-1 as the main protein involved in this type of cell death. The purpose of the study
Correspondence: Dr. Piotr Donizy, Department of Pathomorphology and Oncological Cytology, Wroclaw Medical University, ul. Borowska 213, 50-556 Wroclaw, Poland.

Tel. +48.71.7343964 - Fax: +48.71.7343968.

E-mail: piotrdonizy@wp.pl

Key words: parthanatos, cell death, PARP-1, immunohistochemistry, breast cancer.

Acknowledgments: the study was supported by the grant ST-594 of Wroclaw Medical University.

Conflict of interests: the authors declare no conflict of interests.

Contributions: PD, AH, PS, GP, RM study concepts and design; PD, PS, RM, data acquisition; PD, $\mathrm{RM}, \mathrm{AH}, \mathrm{PS}, \mathrm{CK}, \mathrm{GP}$ data analysis and interpretation; CK, PD statistical analysis; PD, RM manuscript preparation and editing.

Received for publication: 1 July 2013.

Accepted for publication: 1 October 2013.

This work is licensed under a Creative Commons Attribution NonCommercial 3.0 License (CC BYNC 3.0).

(C) Copyright P. Donizy et al., 2013

Licensee PAGEPress, Italy

European Journal of Histochemistry 2013; 57:e35 doi:10.4081/ejh.2013.e35

was to correlate the immunohistochemical parameters of PARP-1 reactivity and the selected cytomorphological features of parthanatos, namely the presence of apoptotic bodies and necrosis in $\mathrm{BC}$ specimens.

\section{Materials and Methods}

\section{Patients}

Tissue samples were obtained from 83 patients treated radically for stage II ductal BC, diagnosed between 1993-1994 in the Lower Silesian Oncology Centre in Wroclaw, Poland. The mean age of the patients was 55.2 years. The patients were selected based on the availability of tissues. All patients underwent surgery (Madden mastectomy) with or without adjuvant treatment. Following the applied treatment, the patients were subjected to permanent control in the Lower Silesia Oncology Centre. The study was approved by the Institutional Review Board of the Wroclaw Medical University, Poland.

\section{Tumor samples and immunohisto- chemistry}

Tumor specimens were fixed in $10 \%$ 
buffered formalin and embedded in paraffin. All haematoxylin and eosin (H\&E) stained sections were examined by two pathologists. One representative slide from tumor was evaluated (the minimal diameter of tumor tissue was 5 $\mathrm{mm}$, maximal was $16 \mathrm{~mm}$ ). Formalin-fixed, paraffin embedded tissue sections were freshly prepared $(4 \mu \mathrm{m})$. Immunohistochemistry was performed as previously described. ${ }^{15-17}$ For the detection of PARP-1, a polyclonal rabbit antibody (clone ab6079; Abcam, Cambridge, UK) was diluted 1:150 in the Antibody Diluent, Background Reducing (DakoCytomation, Gdynia, Poland). The tissue sections were incubated with antibodies for $1 \mathrm{~h}$ at room temperature. Subsequent incubations involved biotinylated antibodies (15 min, room temperature) and a streptavidin-biotinylated peroxidase complex (15 min, room temperature) (LSAB+, HRP, DakoCytomation). NovaRed (Vector Laboratories, Peterborough, UK) was used as a chromogen (10 min, at room temperature). All sections were counterstained with Meyer's haematoxylin. In each case control reactions were included, in which the specific antibody was substituted by a Primary Mouse Negative Control (DakoCytomation).

In classical H\&E staining, three or more apoptotic body per high power field $\mathrm{x} 400$ was defined as a positive case with presence of apoptotic bodies.

\section{Evaluation of immunohistochemical reaction intensity}

The immunohistochemical reaction was estimated independently by two pathologists. Intensity of PARP-1 expression in BC cancer cells was evaluated using a semi-quantitative scale of the ImmunoReactive Score (IRS), ${ }^{18}$ with the author's own modifications, in which the intensity of the colour reaction and the percentage of positive cells were both taken into account. The final, integrated scores ranged from 0-12. Additionally, we observed that normal breast tissue, which was included in some slides was characterized by weak to moderate nuclear-cytoplasmic PARP-1 immunoreactivity. In stromal cells and lymphocytes, nuclear and cytoplasmic PARP-1 staining was also detected. Nevertheless, at the stage of subsequent statistical analyses a two-grade scale system was applied, allocating 0 points for expression of PARP- $1<6$ (low level of PARP-1 immunoreactivity) and 1 for expression of PARP-1 $\geq 6$ (high PARP-1 immunoreactivity). Definition of these two groups and determination of the cut-off point is a specific consensus of histopathological observations and statistical analyses, and of the review of literature concerning PARP-1 expression evaluation.

\section{Statistical analysis}

Statistical analysis was performed using the Statistica 9.1 software package (StatSoft Inc., Tulsa, OK, USA). The exact Fisher was used to analyse associations between PARP-1 protein expression parameters and cytomorphological features of parthanatos. Differences between two groups were tested with the MannWhitney U test. P values $<0.05$ were considered statistically significant.

\section{Results}

\section{Presence of necrosis and apoptotic bodies in breast cancer specimens}

In classical H\&E staining, necrosis was observed in 12 cases (14.5\%) (Figure 1A). Apoptotic bodies were identified in 19 patients (22.9\%) (Figure 1B).
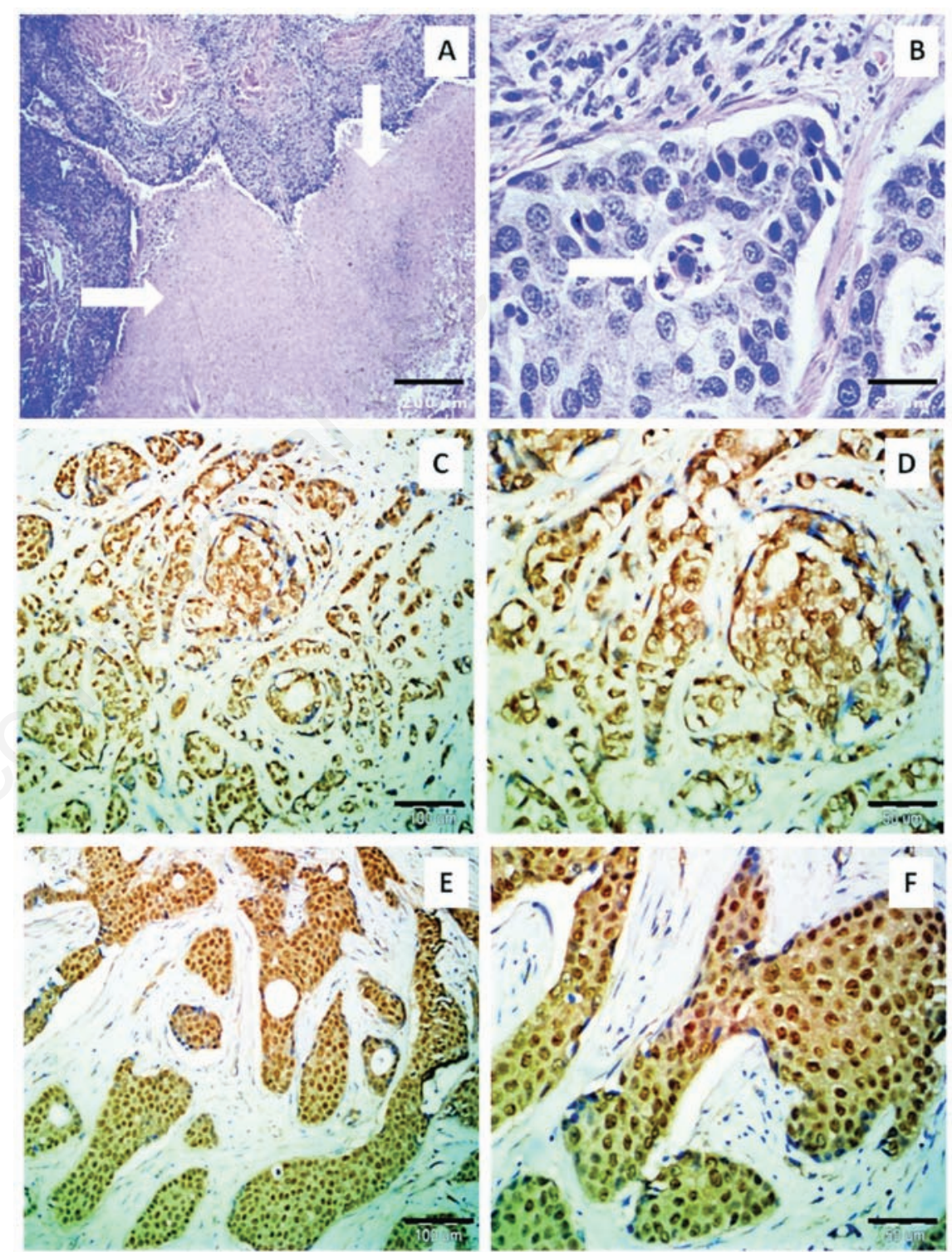

Figure 1. A) Presence of necrosis (arrows) in breast cancer specimen (H\&E; magnification: 40x; scale bar: $200 \mu \mathrm{m})$. B) Apoptotic body formation (H\&E; magnification: 600x; scale bar: $25 \mu \mathrm{m})$. C) Cytoplasmic topography of PARP-1 expression (ImmunoReactive Score (IRS) 9; haematoxylin; magnification: 200x; scale bar: $100 \mu \mathrm{m})$. D) Cytoplasmic expression of PARP-1 in breast cancer cells (IRS 9; haematoxylin; magnification: 400x; scale bar: $50 \mu \mathrm{m})$. E) Nuclear-cytoplasmic topography of PARP-1 expression (IRS 9; haematoxylin; magnification: 200x; scale bar: $100 \mu \mathrm{m})$. F) Nuclear-cytoplasmic expression of PARP-1 in breast cancer cells (IRS 9; haematoxylin; magnification: 400x; scale bar: $50 \mu \mathrm{m})$. 


\section{PARP-1 immunostaining in breast cancer tissue}

PARP-1 expression defined as IRS $>0$ was found in the entire group of 83 patients subjected to investigation. The average IRS was $6.48 \pm 2.5$ and the median was 8 . For the purposes of statistical analysis enhanced immunoreactivity of PARP-1 was defined as IRS $\geq 6$ (55 patients, $66.27 \%$ ), while low immunoreactivity was assigned to IRS values between 0 and 4 (28 patients, 33.73\%).

Histopathological evaluation of the specimens revealed two patterns of PARP-1's sub-cellular localisations. Cytoplasmic localisation alone was observed in 48 cases (57.83\%) (Figure $1 \mathrm{C}, \mathrm{D}$ ), whereas nuclear-cytoplasmic localisation was identified in 35 cases (42.17\%) (Figure 1 E,F).

\section{Correlation between selected cytomorphological features of parthanatos and enhanced PARP-1 immunoreactivity}

High expression of PARP-1 (IRS $\geq 6$ ) was associated with the lack of apoptotic bodies $(\mathrm{P}=0.013)$ and with the absence of necrosis $(\mathrm{P}=0.002)$. Moreover, the presence of apoptotic bodies was correlated with re-distribution of PARP-1 from nucleus to cytoplasm in $\mathrm{BC}$ cells $(\mathrm{P}=0.029)$. Additionally, a tendency was observed between necrosis and loss of nuclear PARP-1 expression $(\mathrm{P}=0.049)$.

\section{Correlation between the lack of apop- totic bodies and the absence of necro- sis, and clinicopathological parame- ters of breast cancer patients}

The lack of apoptotic bodies and the absence of necrosis were correlated with negative status of steroid receptors $(\mathrm{P}=0.000)$. Moreover, the lack of apoptotic bodies and the absence of necrosis were associated with the lack of nodal metastases ( $\mathrm{N}$-) $(\mathrm{P}=0.022$ and $\mathrm{P}=0.041$, respectively) (Table 1 ). Presence of necrosis was correlated with lower age at the moment of $\mathrm{BC}$ diagnosis $(\mathrm{P}=0.037)$. Interestingly, the lack of apoptotic bodies and the lack of necrosis were correlated with higher tumor grading $(\mathrm{P}=0.000)$. We did not observe any significant relationships between overexpression of HER-2, tumor staging according to the Union for International Cancer Control (UICC), type of recurrence and the lack of apoptotic bodies and the lack of necrosis.

\section{Relationship between triple negative immunophenotype of $B C$ and cytomorphological features of parthanatos}

ER and PgR positive status was observed in 61 patients (73.5\%), and HER-2 overexpression

Table 1. Correlation between the lack of apoptotic bodies and the absence of necrosis and clinicopathological parameters of breast cancer patients.

\begin{tabular}{lcc} 
Clinicopathological parameters & Lack of apoptotic bodies & Absence of necrosis \\
ER $^{\circ}$ & 0.000 & 0.000 \\
PgR $^{\circ}$ & 0.000 & 0.000 \\
\hline HER-2 $^{\circ}$ & 0.632 & 0.229 \\
Tumor size (pT) $^{\circ}$ & 0.435 & 0.102 \\
\hline Nodal metastases $(\mathrm{N}+)^{\sharp}$ & 0.022 & 0.041 \\
TNM (UICC)b & 0.493 & 0.211 \\
\hline Grading\# & 0.000 & 0.000 \\
Age $^{\circ}$ & 0.129 & 0.037 \\
Menopause status $^{\#}$ & 0.229 & 0.337 \\
Recurrence $^{\#}$ & 0.542 & 0.475 \\
Adjuvant therapy $^{\sharp}$ & & \\
Chemotherapy & 0.044 & 0.084 \\
Endocrine therapy & 0.556 & 0.598 \\
Radiotherapy & 0.508 & 0.541 \\
\hline
\end{tabular}

TNM, tumor-nodes-metastasis; UICC, Union for International Cancer Control. Statistically significant results $(\mathrm{P}<0.05)$ are in italics. ${ }^{\circ} \mathrm{P}$ value of Mann-Whitney's U test; ${ }^{\sharp}$ v value of Fisher's test.

(in immunohistochemistry) was observed in 19 patients $(22.9 \%)$. We selected a group of triple negative breast cancer (15 cases; $18.1 \%)$ and, surprisingly, we observed that in this group, 9 patients were characterized by low PARP-1 immunoreactivity (IRS <6). In 11 patients (form 15 patients of triple negative phenotype) it was observed only cytoplasmic expression of PARP-1. In this triple negative group, we revealed in 9 patients $(9 / 15 ; 60 \%)$ presence of necrosis and in 10 patients $(10 / 15$; $66.7 \%)$ presence of apoptotic bodies. Due to the small number of patients, we did not perform additional statistical analysis.

\section{Discussion}

In this study we investigated the relationship between enhanced immunoreactivity of PARP-1 in breast cancer cells and selected cytomorphological features of parthanatos, namely the lack of necrosis and the absence of apoptotic bodies in BC specimens. To the best of our knowledge, this is the first clinical analysis of the correlation between PARP-1 expression and morphological features of parthanatos in clinical specimens of BC.

Research into PARP-1-mediated cell death revealed two putative mechanisms which may be responsible for its induction. Initially, a hypothesis of the so-called energetic catastrophe followed by necrotic cell death was proposed. ${ }^{19}$ Adverse effect of enhanced enzymatic activity of PARP-1 in response to DNA damage was attributed to the reduction in the level of $\mathrm{NAD}+$ which is an important cofactor in glycolysis processes and tricarboxylic acid cycle that drive the synthesis of the basic energy substrate ATP. However, further analyses revealed that the decreased intracellular level of NAD+ is by itself not sufficient for initiating cell death. ${ }^{2,20,21}$ The results of recent studies clearly indicate that cell death initiation is closely related with the accumulation of PAR in response to DNA damage. ${ }^{6}$ The polymers function as specific signals and cytophysiological messengers informing about DNA damage that is too extensive to be repaired by the cell itself. Their excess induces parthanatos to prevent and protect against further loss of energy for ineffective DNA repair., ${ }^{6} 7$ Additionally, poly (ADP-ribosyl)ation of cellular proteins might lead to cell death. Kanai et al. ${ }^{22}$ revealed that PARP-1-mediated p53 poly(ADP-ribosyl) ation blocked the interaction of p53 and nuclear export receptor Crml, resulting in the accumulation of p53 in the nucleus. It is worth noting that cytophysiological functions of poly(ADPribosyl)ation of some cellular proteins are still under investigation. It is worth noting that a statistically significant correlation was found related with re-distribution of PARP-1 from the nucleus to the cytoplasm in the presence of apoptotic bodies. It is an important finding which confirms that proper sub-cellular distribution of PARP-1 is essential to ensure its proper biological functioning (in this case parthanatos induction). The analysis of PARP1 subcellular localization revealed that this protein has two patterns of immunohistochemical expression: nuclear-cytoplasmic and only cytoplasmic. It is generally assumed that PARP-1 is a nuclear protein, able to detect and bind damaged DNA. The finding of a fraction of PARP-1 within the cytoplasm concomitant with the appearance of apoptotic bodies needs a 
careful discussion, possibly considering that a proteolytic fragment of PARP-1 is extruded from the nucleus in the final steps of caspasedependent apoptosis. ${ }^{23,24}$ We need to emphasize that in clinical material from $\mathrm{BC}$ patients two scientific groups observed two different patterns of PARP-1 expression in BC cells. Von Minckwitz et al..$^{25}$ described predominant cytoplasmic immunoreactivity, on the other hand, Rojo et al. ${ }^{26}$ observed only nuclear expression (without cytoplasmic topography). Due to contradictory results, further studies are necessary to fully understand and describe proper pattern of PARP-1 expression.

The present brief note is an attempt at showing and confirming, with the use of clinical material obtained from $\mathrm{BC}$ patients, that strong correlations exist between overexpression of PARP-1 which is the key parthanatos inducing protein, and relatively simple (observed during a routine histopathological evaluation) cytomorphological features of this specific form of cell death (absence of necrosis and apoptotic bodies). It must be clearly stressed that immunohistochemistry cannot substitute for sophisticated techniques of molecular biology in exploring the mechanism of induction and course of parthanatos, yet the present paper is an interesting voice in the discussion on this extremely intriguing form of cell death. Furthermore, due to contradictory results and unclear correlations, further studies are necessary to fully understand the mystery of parthanatos.

\section{References}

1. Javle M, Curtin NJ. The role of PARP in DNA repair and its therapeutic exploitation. Br J Cancer 2011;105:1114-22.

2. Kiliańska ZM, Żołnierczyk J, WęsierskaGądek J. Biological activity of poly(ADPribose) polymerase-1. Postepy Hig Med Dosw 2010;64:344-63.

3. Krishnakumar R, Kraus WL. The PARP side of the nucleus: molecular actions, physiological outcomes, and clinical targets. Mol Cell 2010;39:8-24.

4. Horton JK, Wilson SH. Hypersensitivity phenotypes associated with genetic and synthetic inhibitor-induced base excision repair deficiency. DNA Repair 2007;6:530-43.

5. Ame JC, Spenlehauer C, de Murcia G. The PARP superfamily. Bioessays 2004;26:882-93.
6. Wang Y, Kim NS, Haince JF, Kang HC, David KK, Andrabi SA, et al. Poly(ADPribose) (PAR) binding to apoptosis-inducing factor is critical for PAR polymerase-1dependent cell death (parthanatos). Sci Signal 2011;4:ra20.

7. Wang Y, Dawson VL, Dawson TM. Poly(ADP-ribose) signals to mitochondrial AIF: a key event in parthanatos. Exp Neurol 2009;218:193-202.

8. Delettre C, Yuste VJ, Moubarak RS, Bras M, Lesbordes-Brion JC, Petres S, et al. AIFsh, a novel apoptosis-inducing factor (AIF) pro-apoptotic izoform with potential pathological relevance in human cancer. $\mathrm{J}$ Biol Chem 2006;281:6413-27.

9. Yu SW, Wang H, Poitras MF, Coombs C, Bowers WJ, Federoff HJ, et al. Mediation of poly(ADP-ribose) polymerase-1-dependent cell death by apoptosis-inducing factor. Science 2002;297:259-63.

10. Wang H, Yu SW, Koh DW, Lew J, Coombs C, Bowers W, et al. Apoptosis-inducing factor substitutes for caspase executioners in NMDA-triggered excitotoxic neuronal death. J Neurosci 2004;24:10963-73.

11. Kroemer G, El-Deiry WS, Golstein P, Peter ME, Vaux D, Vandenabeele $P$, et al. Classification of cell death: recommendations of the Nomenclature Committee on Cell Death. Cell Death Differ 2005;12: n1463-7.

12. Edinger AL, Thompson CB. Death by design: apoptosis, necrosis and autophagy. Curr Opin Cell Biol 2004;16:663-9.

13. Galia A, Calogero AE, Condorelli R, Fraggetta F, La Corte A, Ridolfo F, et al. PARP-1 protein expression in glioblastoma multiforme. Eur J Histochem 2012;56:e9.

14. Salemi M, Galia A, Fraggetta F, La Corte C, Pepe P, La Vignera S, et al. Poly (ADPribose) polymerase 1 protein expression in normal and neoplastic prostatic tissue. Eur J Histochem 2013;57:e13.

15. Halon A, Materna V, Drag-Zalesinka M, Nowak-Markwitz E, Gansukh T, Donizy P, et al. Estrogen receptor alpha expression in ovarian cancer predicts longer overall survival. Pathol Oncol Res 2011;17:511-8.

16. Halon A, Nowak-Markwitz E, Maciejczyk A, Pudelko M, Gansukh T, Györffy B, et al. Loss of estrogen receptor beta expression correlates with shorter overall survival and lack of clinical response to chemotherapy in ovarian cancer patients. Anticancer Res 2011;31:711-8.
17. Szczuraszek K, Halon A, Materna V, Mazur G, Wrobel T, Kuliczkowski K, et al. Elevated YB-1 expression is a new unfavorable prognostic factor in non-Hodgkin's lymphomas. Anticancer Res 2011;31:2963-70.

18. Remmele W, Stegner HE. Recommendation for uniform definition of an immunoreactive score (IRS) for immunohistochemical estrogen receptor detection (ER-ICA) in breast cancer tissue. Pathologe 1987;8:138-40.

19. Bernstein C, Bernstein H, Payne CM, Garewal H. DNA repair/proapoptotic dualrole proteins in five major DNA repair pathways: fail-safe protection against carcinogenesis. Mutat Res 2002;511:145-78.

20. Fossati S, Cipriani G, Moroni F, Chiarugi A. Neither energy collapse nor transcription underline in vitro neurotoxicity of poly(ADP-ribose) polymerase hyper-activation. Neurochem Int 2007;50:203-10.

21. Moubarak RS, Yuste VJ, Artus C, Bouharrour A, Greer PA, Menissier-de Murcia J, et al. Sequential activation of poly(ADP-ribose) polymerase 1, calpains, and Bax is essential in apoptosis-inducing factor-mediated programmed necrosis. Mol Cell Biol 2007;27:4844-62.

22. Kanai M, Hanashiro K, Kim SH, Hanai S, Boulares AH, Miwa M, et al. Inhibition of Crm1-p53 interaction and nuclear export of p53 by poly(ADP-ribosyl)ation. Nat Cell Biol 2007;9:1175-83.

23. Soldani C, Lazzè MC, Bottone MG, Tognon G, Biggiogera M, Pellicciari CE, et al. Poly(ADP-ribose) polymerase cleavage during apoptosis: when and where? Exp Cell Res 2001;269:193-201.

24. Soldani C, Scovassi AI. Poly(ADP-ribose) polymerase-1 cleavage during apoptosis: an update. Apoptosis 2002;7:321-8.

25. von Minckwitz G, Müller BM, Loibl S, Budczies J, Hanusch C, Darb-Esfahani S, et al. Cytoplasmic poly(adenosine diphosphate-ribose) polymerase expression is predictive and prognostic in patients with breast cancer treated with neoadjuvant chemotherapy. J Clin Oncol 2011;29:2150-7.

26. Rojo F, Garcia-Parra J, Zazo S, Tusquets I, Ferrer-Lozano J, Menendez $\mathrm{S}$, et al. Nuclear PARP-1 protein overexpression is associated with poor overall survival in early breast cancer. Ann Oncol 2012;23: 1156-64. 